\title{
A Parameters Calibration Method in Simulated Complex Traffic Network
}

\author{
Hu Xinghua ${ }^{1,2, *}$ and Zhang $\mathrm{Yu}^{2}$ \\ ${ }^{I}$ School of Traffic and Transportation, Beijing Jiaotong University, Beijing 10044, China \\ ${ }^{2}$ Chongqing Communications Planning Survey and Design Institute, Chongqing 401121, China
}

\begin{abstract}
Traffic simulation models have been extensively used because of their ability to model the dynamic stochastic nature of transportation systems. Parameter calibration is very complex and does not give optimal results easily. Besides, it is also time-consuming especially for large and complex networks. Initially, the procedure of traffic micro-simulation parameter calibration was put forward. A Vehicle Intelligent Simulation Software Model (VISSIM) models were selected for parameter calibration in complex-network, and, the role of Simultaneous Perturbation Genetic Algorithm (SPGA) was examined in the optimization of component. Moreover an automatic calibration methodology for micro-simulation models was developed in order to select the best parameter set based on the observed Intelligent Transportation Systems (ITS) data which proved effective for different networks. Finally, the methodology was applied to calibrate the Beijing city VISSIM models, followed by the comparison of convergence rate of Genetic Algorithm (GA), Simultaneous Perturbation Stochastic Approximation (SPSA) and SPGA algorithm. The results show that the SPGA was effective and had good performance.
\end{abstract}

Keywords: Complex network simulation, parameter calibration, stochastic approximation genetic algorithm, traffic engineering.

\section{INTRODUCTION}

With the gradual implementation of Intelligent Transportation System (ITS), the traffic micro-simulation models have been used extensively in China. Traffic microsimulation models use a large number of independent parameters to describe the traffic system operation, traffic flow characteristics and drivers' behaviour; and its parameters values have a great impact on simulation results. Therefore, in practice, the simulation parameters must be calibrated by considering the traffic characteristics of the simulation network to ensure the validity of the simulation model. However, the parameter calibration of traffic micro-simulation models is a complex and systematic work, especially for the large and complex network whose simulation environment is complex and with a lot of parameters to be calibrated. In addition it is time-consuming and difficult to find the optimal solution with artificial means. The literature [1-7] made a research on parameters calibration of traffic simulation model with Genetic Algorithm (GA) and Simultaneous Perturbation Stochastic Approximation (SPSA), and drew a conclusion of existed methods of parameters calibration. However, GA is inefficient in local search, slow and timeconsuming in convergence rate of parameter calibration. SPSA has a higher efficiency in local search. But it is hard to seek out the optimal solution. In this paper, with VISSIM simulation model as the basic platform, the parameter calibration methods and optimization algorithms for large-scale simulation network are studied, the parameter calibration (SPGA) simulation model was established, and applied for

*Address correspondence to this author at the Beijing Jiaotong University, No.3 Shangyuancun, Beijing, China; Tel: +86-23-88913869; Fax: +86-23-88913869; E-mail: bjtufox@163.com parameter calibration of large-scale simulation network in Beijing. By comparing the convergence rate between GA and SPSA, the superiority of SPGA was verified.

In the following sections, parameter calibration method firstly is introduced, including parameter selection, objective function setting and parameter calibration process. The third part introduces the parameter calibration algorithm, and then a example is application. Finally, the results are presented followed by the conclusion.

\section{PARAMETER CALIBRATION METHOD OF TRAFFIC SIMULATION MODEL}

\subsection{Selection of Calibration Parameters}

In the parameter calibration of traffic simulation models, the drivers' behaviour parameters could affect the accuracy of the simulation results directly. This paper selected ten key parameters of the drivers' behaviours according to the VISSIM model characteristics, and the name, default value and the range of the parameter are shown in Table 1.

\subsection{Targeted Function of Calibration}

The goal of calibration of model parameters is to match model simulation results with experimental results in maximum. In this paper, with the traffic flow as its target, the following function is used to evaluate the convergence accuracy of the model:

$$
F=\sum_{i=1}^{M} G E H\left(v_{i}\right)+G E H\left(f_{i}\right)
$$


Table 1. Calibration parameters.

\begin{tabular}{|c|c|c|c|}
\hline Name of Parameters & Default & Range & Unit \\
\hline Waiting Time before Disappeared & 60.00 & $30 \sim 90$ & Second \\
\hline Minimum Space Headway & 0.50 & $0.1 \sim 1.0$ & Metre \\
\hline Maximum Deceleration & -4.00 & $-6 \sim-2$ & $\mathrm{M} / \mathrm{S}^{2}$ \\
\hline$-1 \mathrm{Metre} / \mathrm{S}^{2} /$ Distance & 100.00 & $50 \sim 150$ & Metre \\
\hline Acceptable Deceleration & -1.00 & $-3 \sim-0.2$ & $\mathrm{M} / \mathrm{S}^{2}$ \\
\hline Maximum Vehicle Visibility Distance & 250.00 & $200 \sim 300$ & Metre \\
\hline Average Parking Space & 2.00 & $0 \sim 5$ & Metre \\
\hline Additional Part of the Safe Distance & 2.00 & $0.2 \sim 5$ & \\
\hline Multiples of Some Safe Distance & 3.00 & $0.2 \sim 5$ & \\
\hline Minimum Transverse Distance on $50 \mathrm{Km} / \mathrm{h}$ & 1.00 & $0 \sim 6$ & Metre \\
\hline
\end{tabular}

$M:$ the number of detectors;

$v_{i}$ : the speed of the $i$-th test data of one-way point;

$f_{i}$ : cross-section flow of the $i$-th test data of one-way point .

Statistical method of $G E H\left(V_{p}, V_{m}\right)$ is defined as the following:

$\operatorname{GEH}\left(v_{p}, v_{m}\right)=\sqrt{\frac{\left(V_{p}-V_{m}\right)^{2}}{\left(V_{p}+V_{m}\right) / 2}}$

$V_{p}:$ Model simulation values;

$V_{m}:$ Measured value.

When there are more than $85 \% G E H\left(V_{p}, V_{m}\right)$ value is smaller than 5, model calibration results are acceptable.

\subsection{Parameter Calibration Process}

According to the basic principles of SPGA, the process of the parameters calibration established in this paper is shown in Fig. (1).

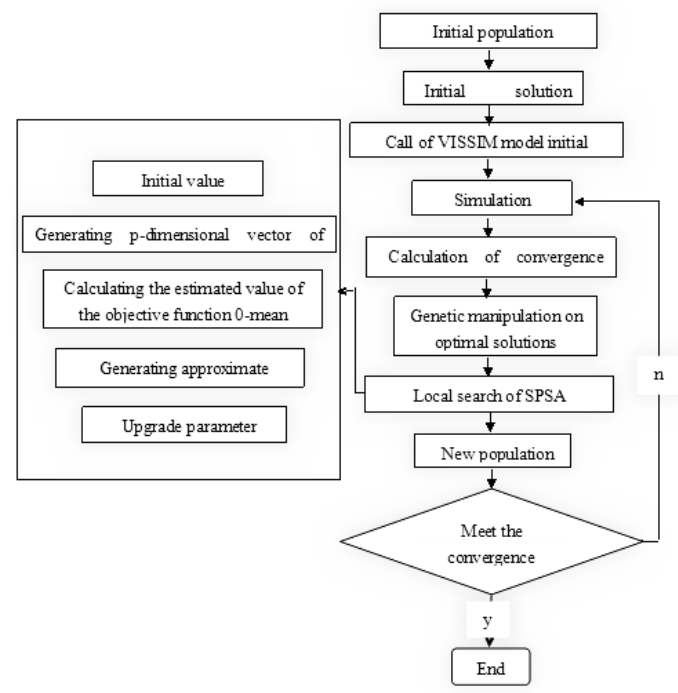

Fig. (1). Flow chart of calibration procedure based on SPGA.

\section{THE REALIZATION PARAMETERS CALIBRA- TION BASED ON SPGA ALGORITHM}

The method of SPGA parameters calibration is proposed by combining the deficiency of parameters calibration in GA and SPSA in large-scale traffic simulation model, and by improving the SPSA algorithm. SPGA's basic idea is to use genetic algorithm to generate the initial population and do genetic manipulation to it, to use the SPSA algorithm to locally search species that generated in every step of the genetic manipulation. Then, SPSA will produce a new generation of population to replace the current generation population. If the result meet the goal, then stop the search and output all the most optimal solutions produced by this process and the current generation population, else, make the next generation [8]. In this paper, the automation of parameters calibration was realized by using the VISSIM model with abundant external program interface and by hybrid programming of $\mathrm{C \#}$ and Matlab. And the steps of parameters calibration based on SPGA are as follows:

Step 1: Initialization and parameter selection. This step determined population size, hybrid probability, mutation probability, variable dimensions, the parameter values of the upper and lower limits, the maximum genetic algebra and local search parameters of $a, c, A, \alpha$ and $\gamma$, generating the initial population with the genetic algebra gen $=0$;

Step 2: The initial population generated was entered into VISSIM simulation model to calculate the objective function value - FitnV of individuals;

Step 3: Values of fitness were distributed, and by selection, recombination, and mutation, sub-generation was obtained; $M$-individual was chosen from the collection and $R$ -individual was selected from previous generations to form the intermediate species;

Step 4: Parameters composed by intermediate species were assigned to $\theta_{0}$, making the counter $k=0$, generate independent random perturbation vector $\Delta_{k}$ of 0 -meaned and $\mathrm{p}$ dimension randomly, with $\Delta_{k}$ containing three elements$\Delta_{k i}, i=1,2,3$, with the value being: 
$\Delta_{k i}\left\{\begin{array}{l}1, \text { probability }=1 / 2 \\ -1, \text { probability }=1 / 2\end{array}\right.$

Step 5: Two sets of perturbation parameters were defined. According to the perturbation parameter $\Delta_{k}$ of step 4 , two sets of perturbation parameters were defined, respectively to operate simulation model and calculate the estimated FitnV of the objective function;

Step 6: Close gradient value of the objective function was calculated by simulation results, and the calibration parameters- $\hat{\theta}_{k}$ were upgraded and re-inserted to generate new population;

Step 7: Steps 2 to 6 were repeated until the result meets the requirement. 1 was added to gen in each loop, until the objective function met the convergence conditions.

\section{EXAMPLE APPLICATION}

The selected test area was the fast three-ring road area of Beijing, including an expressway of length $18 \mathrm{~km}$, with a number of main and branch roads, seven overpasses, and 108 bus lines. Traffic flow data sources detectors measured microwave remote sensing data from 7:00 to 9:00 during the morning in October 25, 2010. Data collected by the detector included traffic flow, speed, share rate, and the acquisition time of 24 hours. Test area network is shown in Fig. (2).

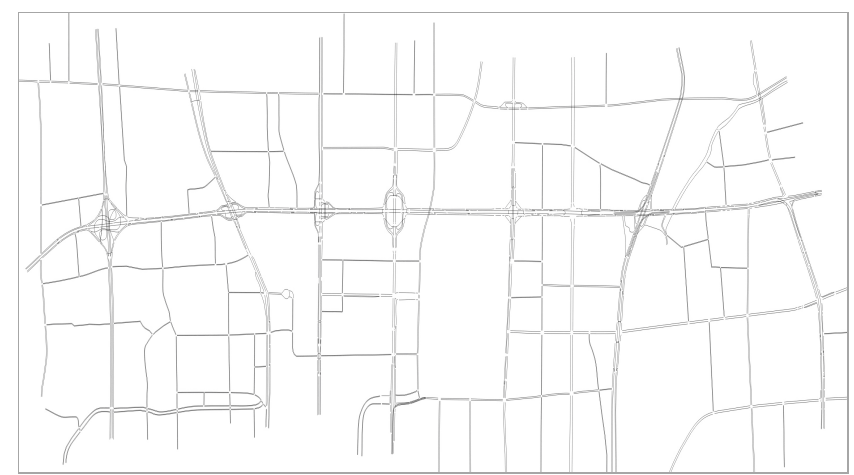

Fig. (2). Test network.

After building the platform of VISSIM simulation model, GA, SPSA and SPGA were used to estimate ten driver behavior parameters. After iterating 251 times, 172 times and 146 times with GA, SPSA and SPGA respectively, the algorithm objective function value converged to 631,544 and 185, respectively. Target change process is shown in Fig. (3).

The following Table 2 shows the optimized value of each parameter after calibration.

In order to verify the validity of the model, calibrated parameters were used to perform model simulation and analyze the correlation between the output and measured values of simulation model, as shown in Fig. (4) and Fig. (5) $[9,10]$.

As shown in Fig. (4) and Fig. (5), in SPGA, the correlation between the coefficient of simulation flow and measured flow was 0.86 and 0.92 respectively, which shows that the result was acceptable. Moreover, SPGA has a lower conver- gence value than the GA and SPSA algorithms, which shows that has better accuracy.

Table 2. Parameter calibration value.

\begin{tabular}{|c|c|c|}
\hline Name of Parameters & Default & $\begin{array}{c}\text { SPGA Optimized } \\
\text { Value }\end{array}$ \\
\hline \hline Waiting Time before Disappeared & 60 & 46 \\
\hline Minimum Space Headway & 0.5 & 0.2 \\
\hline Maximum Deceleration & -4 & -3.5 \\
\hline -1Metre/S $/$ Distance & 100 & 69 \\
\hline Acceptable Deceleration & -1 & -2.4 \\
\hline Maximum Vehicle Visibility Distance & 2 & 287 \\
\hline Average Parking Space & 250 & 1.36 \\
\hline Additional Part of the Safe Distance & 2 & 3.37 \\
\hline Multiples of Some Safe Distance & 2 & 2.29 \\
\hline Minimum Transverse Distance on $50 \mathrm{~km} / \mathrm{h}$ & 3 & \\
\hline
\end{tabular}

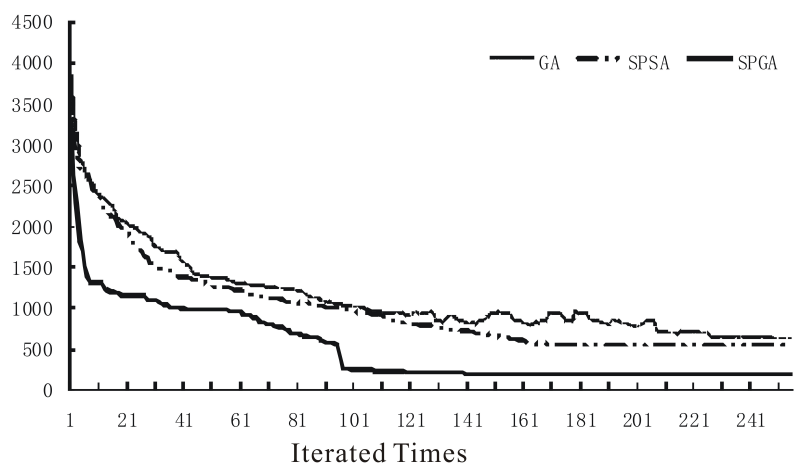

Fig. (3). Comparison of the algorithm convergence speed.

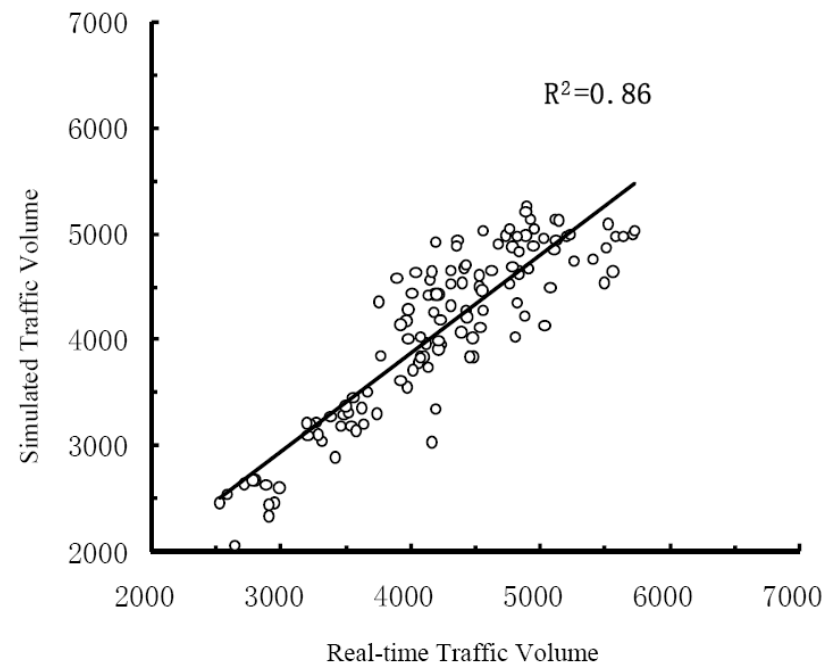

Fig. (4). Comparison of observed and simulated flows with SPGA. 


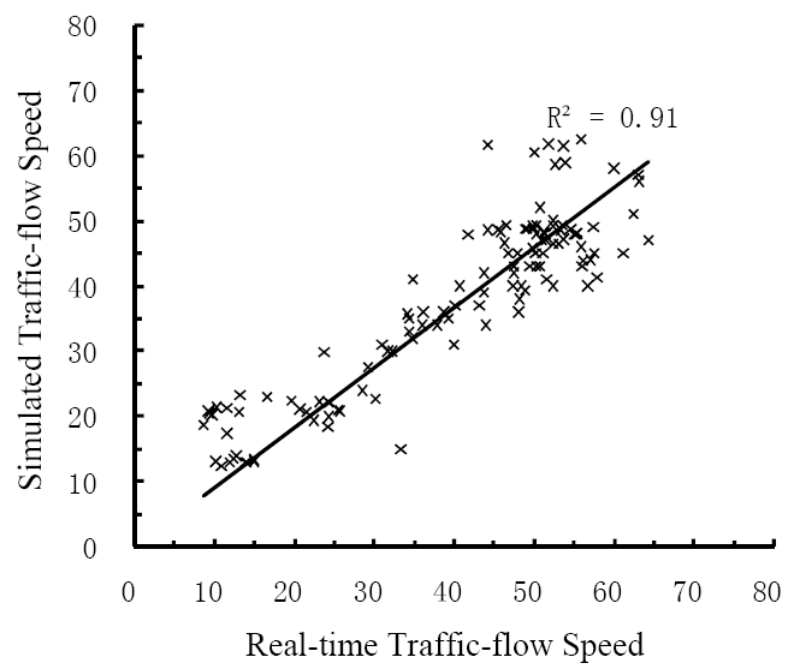

Fig. (5). Comparison of observed and simulated flows with SPGA.

\section{CONCLUSION}

This paper studied parameters calibration method and the process of microscopic simulation mode in large-scale network environment, and proposed the formation of parameter calibration of SPGA algorithm combined with $\mathrm{C \#}$ and Matlab programming language to achieve automation of parameters calibration. The method was applied to the calibration of driver behaviour parameters of VISSIM simulation model in large areas of Beijing expressway. The results show that compared with GA and SPSA, the convergence rate of large-scale network simulation parameters calibration method based on SPGA was 1.7 times and 1.2 times faster respectively, which greatly reduced the time of parameters calibration and provided a new way for parameters calibration of large-scale network simulation model. At the same time, traffic flow and speed were tested after parameters calibration, and the results show that the error was within an acceptable range and the error with SPGA was smaller, which proves the advantages and feasibility of SPGA for parameter calibration of microscopic traffic simulation model of large-scale networks.

\section{CONFLICT OF INTEREST}

The authors confirm that this article content has no conflict of interest.

\section{ACKNOWLEDGEMENTS}

This work is supported by the Major Project of the National Natural Science Foundation, China (No. 71390332) and the National Natural Science Foundation, China (No. 60870014).

\section{REFERENCES}

[1] S. Jian, Y. Xiaoguang, and L. Hao-de, "The research on parameters correction of microscopic traffic simulation system," Journal of System Simulation, vol. 19, no. 1, pp. 48-50, 2007.

[2] B. B. Park, and H. Qi., "Development and evaluation of a calibration and validation procedure for microscopic simulation models," Virginia Transportation Research Council, 2004.

[3] L. Chu, "A calibration procedure for microscopic traffic simulation," Annual Meeting CD-ROM, transportation research board. Washington, D.C., 2004.

[4] B. Park, and H. Qi, "Development and evaluation of a calibration and validation procedure for microscopic simulation models. VTRC 05-CR1," Virginia Transportation Research Council, Charlottesville, 2004.

[5] J.B. Lee, and K. Ozbay, "Calibration of a macroscopic traffic simulation model using enhanced simultaneous perturbation stochastic approximation", Methodology, Washington, D.C., $87^{\text {th }}$ Transportation Research Board Annual Meeting, 2008.

[6] R. E. Benekohal, "Procedure for validation of microscopic traffic flow simulation models," Washington, D.C, $70^{\text {th }}$ Transportation Research Board Annual Meting, 1991.

[7] J.C. Spall, "Implementation of the simultaneous perturbation algorithm for stochastic optimization," IEEE Transactions on Aerospace and Electronic Systems, vol. 34, no. 3, pp. 817-823,1998.

[8] L. Xiujuan, "Stochastic gradient ga algorithm of multi-objective optimization problem," Journal of Nanjing Aeronautics and Astronautics University, vol. 35, no. 4, pp. 455-458, 2003.

[9] L. Wenhao, "The application of genetic algorithms in estimation of important parameters in system dynamics model," Journal of Fujian Agriculture and Forestry University, 2002, vol. 31, no. 3, pp. 404-407, 2002.

[10] Z. Yu, Y. Lei, Z. Nale, Z. Liying, and C. Xumei, "Application of simultaneous perturbation stochastic approximation algorithm in parameter calibration of vissim microscope simulation model," Journal of Transportation Systems Engineering and Information Technology, vol. 10, no. 4, pp. 44-49, 2010.

Received: May 26, 2015
(C) Xinghua and Yu; Licensee Bentham Open.

Revised: July 14, 2015

Accepted: August 10,2015

This is an open access article licensed under the terms of the (https://creativecommons.org/licenses/by/4.0/legalcode), which permits unrestricted, noncommercial use, distribution and reproduction in any medium, provided the work is properly cited. 